\title{
FTIR Study of Multifunctional Coatings
}

\author{
C. Paluszkiewicz ${ }^{a, *}$, E. Deugon ${ }^{a}$ And W.M. KwiateK ${ }^{b}$ \\ ${ }^{a}$ Faculty of Materials Science and Ceramics, AGH - University of Science and Technology \\ al. A. Mickiewicza 30, 30-059 Kraków, Poland \\ ${ }^{b}$ The Henryk Niewodniczański Institute of Nuclear Physics, Polish Academy of Sciences \\ E. Radzikowskiego 152, 31-342 Kraków, Poland
}

\begin{abstract}
Nanoparticles of hydroxyapatite (HAp) or tricalcium phosphates (TCP) connected with fibrous phase create biomimetic system between the natural fibrous and ceramic materials building a bone. Chemical bonding between the implant and host tissue takes place through the phosphate layer, which is created on the bioactive implant surface when in contact with the body fluids environment. The Fourier transform infrared spectroscopy can yield microstructural information on the segment level complementary to the morphological information acquired from X-ray scattering as well as electron microscopy. The Fourier transform infrared method is applied to study thin films on different substrates. Moreover, the Fourier transform infrared microscope technique allows to obtain surface and cross-section maps in reflection and transmission modes. This leads to visualization of chemical imaging between substrates and films. In this work, the coatings with different addition of nanohydroxyapatite were deposited by electrodeposition method on titanium and Ti6Al4V alloys. Additionally, sublayers, such as $\mathrm{TiO}_{2}$, were used to increase hydroxyapatite coating adhesion. The selection of suspension composition, depositing time and layer heat treatment conditions have the conclusive influence on the films parameters. All these experimental parameters were monitored during the sample preparation procedure. Changes in phase composition of biomaterials were determined by the Fourier transform infrared reflection technique based on focal plane array detection system. It has been found that results obtained by the Fourier transform infrared spectroscopy show the differences between the studied samples as well as that optimum time of HAp deposition was $90 \mathrm{~s}$.
\end{abstract}

PACS: 82.80.Gk, 81.15.Pq, 68.37.Yz, 82.80.Dx, 78.30.Er

\section{Introduction}

Bio-ceramic materials have revolutionized orthopedic and dental repair of damaged parts of the bone system. The bio-inert ceramic materials show attractive properties, such as strength and fracture for medical applications. Some of biomaterials, due to their biocompatibility, allow manipulation and adaptation to the shape and dimensions of bone defects [1-3]. Titanium and its alloys are the materials which are widely applied for the fabrication of bio-implants. The nature of their surfaces can directly influence cellular response, ultimately affecting the rate and quality of new tissue formation. On the other hand, this kind of surface influences also the adhesion between ceramic cement and metal. In order to improve titanium implants properties, they are usually covered with various kinds of ceramic layers [4-8]. Hydroxyapatite coated implants are being used clinically in orthopedics and dental fields. It has attracted interest for biological implants due to its biocompatibility. Solgel and electrophoretic methods are the most popular ways applied to modify metal surfaces [7-10].

Infrared and the Raman spectroscopy methods are valuable tools in the biomaterials engineering allowing the study of the processes occurring during their prepa-

* corresponding author; e-mail: cpalusz@agh.edu.pl ration. The Fourier transform infrared (FTIR) spectroscopy has been extensively used for in vitro and in vivo investigations of degradation mechanism and kinetics of different biomedical devices. It has been also used to characterize the crystalline and amorphous domains in bio-mineralization process $[3,7,9,11-13]$.

In this work the coatings with different addition of nanohydroxyapatite were electrodeposited on titanium and Ti6Al4V alloys. Furthermore, sublayers, such as $\mathrm{TiO}_{2}$, were used to increase apatite coating adhesion. Additionally, this layer was enriched by nanosilver, because $\mathrm{Ag}^{+}$has a strong antibacterial activity $[14,15]$. The selection of suspension composition, depositing time and layer heat treatment conditions have the conclusive influence on the films parameters. All these experimental parameters were monitored during the sample preparation procedure.

\section{Materials and methods}

In this work a two-step process of titanium surface modification was used. Thus, metal samples in the form of rectangular plates $10 \times 15 \mathrm{~mm}^{2}$ were prepared from titanium and its alloy Ti6Al4V. The metal plates were mechanically and chemically cleaned and then their surfaces were coated using the sol-gel technique. In this method, the layers were obtained by withdrawing the metal samples at a constant speed from titanium (Ti) 
sols. The sols were prepared from solutions containing $\mathrm{Ti}\left(\mathrm{OC}_{3} \mathrm{H}_{7}\right)_{4}, \mathrm{C}_{2} \mathrm{H}_{5} \mathrm{OH}, \mathrm{CH}_{3} \mathrm{COOH}$ and $\mathrm{H}_{2} \mathrm{O}$. Thickness of the layers was checked by multiple dipping and withdrawing of the samples from the sol. Next the samples were dried and heated at $500{ }^{\circ} \mathrm{C}$ in argon atmosphere.

In the second step, the layers obtained on the metal were covered by nanohydroxyapatite using electrophoretic deposition. In this case titanium alloys with precoatings were used as the cathodes and immersed in the electrophoretic tank (containing ethanol and HAp produced by Chema-Elektromet Co., Rzeszów) with the suspension for HAp deposition. Additionally, nano Ag particles were added in order to increase bactericidity of the material. Experiments were performed at room temperature at a constant voltage of $30 \mathrm{~V}$ and deposition time from 10 to $180 \mathrm{~s}$. The distance between cathode and anode was $20 \mathrm{~mm}$.

After each step of preparation, metal surface modification was analyzed by FTIR spectroscopy. FTIR measurements of the coatings were performed using reflection technique. All spectra were measured at $4 \mathrm{~cm}^{-1}$ resolution. The FTIR spectra were collected using Bruker system OPUS-65. Microscope (Hyperion-3000) with a $15 \times$ Cassegrain objective was equipped with mercury cadmium telluride (MCT) and focal plane array (FPA) $(64 \times 64$ pixels $)$ detectors and $x y$-stages. The video camera attached to the microscope enabled optical imaging of the investigated area. Spatial resolution was about $3 \mu \mathrm{m}$. In the case of FTIR imaging, calculations of $\mathrm{PO}_{4}^{3-}$ band areas were performed in the range from $900 \mathrm{~cm}^{-1}$ to $1200 \mathrm{~cm}^{-1}$

Scanning electron microscopy (SEM) images of microstructure and energy dispersion X-ray (EDX) analysis of layers on metal substrates were performed using scanning electron microscope Phillips XL 30 with an energy dispersive X-ray analyzer LINK ISIS-EDX.

\section{Results}

Figure 1 shows examples of characteristic microscopic FTIR spectra after background subtractions of hydroxyapatite layers which were deposited on $\mathrm{Ti}$ surface for different time intervals: (a) $10 \mathrm{~s}$, (b) $20 \mathrm{~s}$, (c) $30 \mathrm{~s}$, (d) $60 \mathrm{~s}$, (e) $90 \mathrm{~s}$. The results for $120 \mathrm{~s}$ and $180 \mathrm{~s}$ of deposition time are not presented because after such deposition time the HAp layers were too thick and fall off. Each spectrum was recorded in reflection mode through a $250 \mu \mathrm{m} \times 250 \mu \mathrm{m}$ aperture using MCT detector. The increase in intensity and areas of the bands corresponding to phosphate groups along with time of HAp layers deposition is seen. The major vibrational bands in these spectra are due to stretching vibrations $\nu_{3}$ at $1032 \mathrm{~cm}^{-1}$ and $1093 \mathrm{~cm}^{-1}$ and $\nu_{1}$ at $965 \mathrm{~cm}^{-1}$ corresponding to $\mathrm{PO}_{4}^{3-}$ groups [11-13].

It is recognized that FTIR microspectroscopy with FPA detection gives more detailed information from the analyzed area than that using the MCT detector. In this case each element of detector is used to generate a spectrum corresponding to a separate location of the sample.

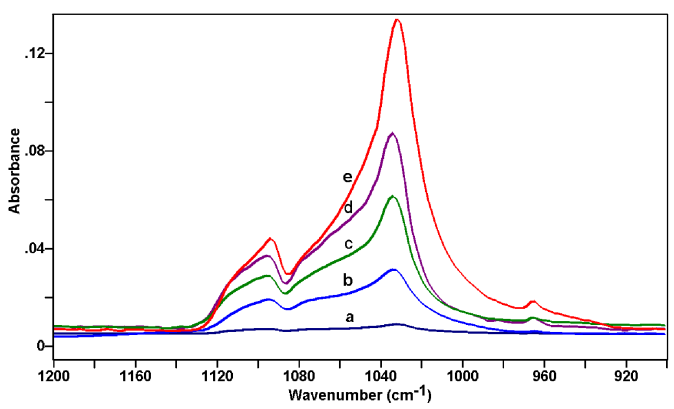

Fig. 1. The average FTIR microreflection spectra of Ti surface $\left(250 \times 250 \mu \mathrm{m}^{2}\right)$ after different time of HAp layers deposition: (a) $10 \mathrm{~s}$, (b) $20 \mathrm{~s}$, (c) $30 \mathrm{~s}$, (d) $60 \mathrm{~s}$, (e) $90 \mathrm{~s}$.

FPA detector with $64 \times 64$ elements allows to receive 4096 spectra in a short time. It makes it possible to obtain information from the area below $10 \times 10 \mu \mathrm{m}^{2}$ square. There are few ways to create chemical images which give various information. One of the approach is to use a single data from each pixel to create an intensity map, which will represent the intensity at a single wavelength or the integrated intensity of an absorption band.

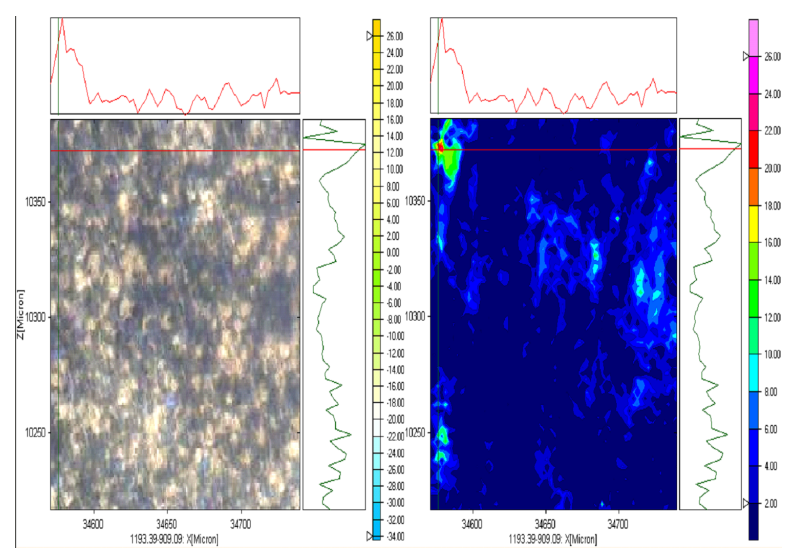

Fig. 2. Optical view and chemical image after $10 \mathrm{~s}$ of HAp deposition on Ti surface.

Figures 2, 3, and 4 show microscopic view (left) and FTIR images of chemical mapping for analyzed surface ceramic layers. The intensity values of the stretching $\mathrm{PO}_{4}^{3-}$ vibrations near $1030 \mathrm{~cm}^{-1}$ are displayed as a function of $x, y$ position (right). In infrared maps the red and blue regions indicate maximum and minimum of integrated absorbance values, respectively. The results indicate existence of differences in phosphate distribution on the surface of metals.

The results obtained after $10 \mathrm{~s} \mathrm{HAp}$ deposition indicate only few points of grains containing phosphate groups (Fig. 2). Differences in phosphate distribution can influence biocompatibility of ceramic implants. More uniform distribution of HAp in mapped areas can be seen in Figs. 3 and 4. Following FTIR analysis one can conclude 


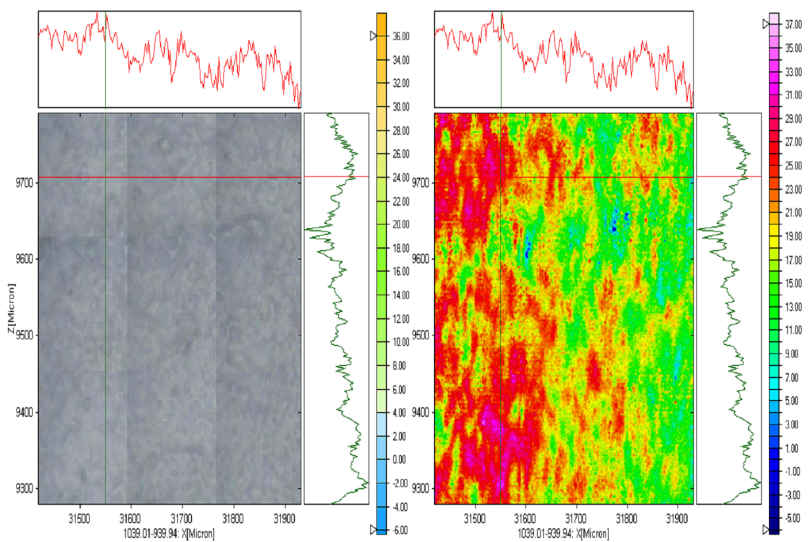

Fig. 3. As in Fig. 2, but after $30 \mathrm{~s}$ of HAp deposition.

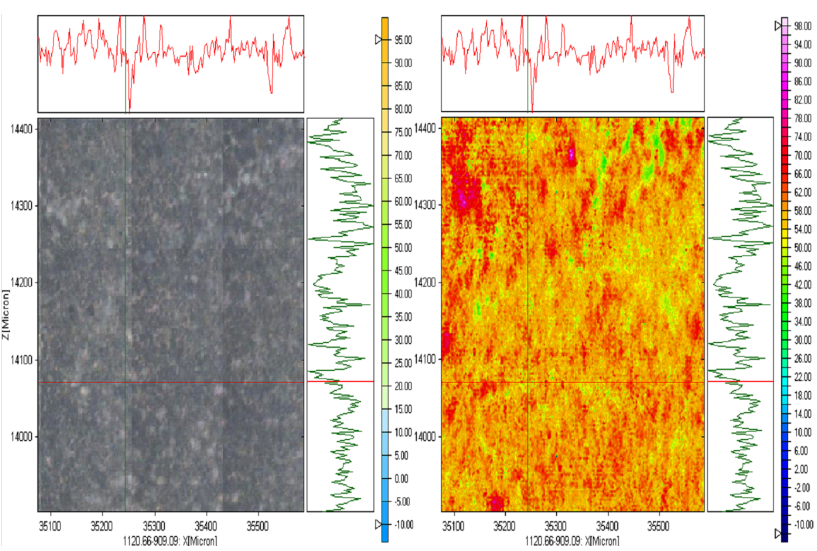

Fig. 4. As in Fig. 2, but after $90 \mathrm{~s}$ of HAp deposition.

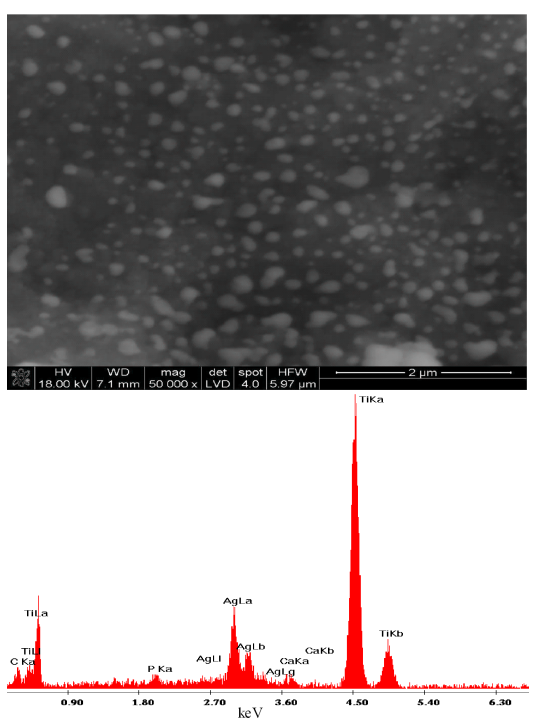

Fig. 5. Example of SEM image and X-ray fluorescence spectrum of EDX analysis of HAp layers with nano Ag particles deposited on titanium alloys (deposition time $10 \mathrm{~s})$.
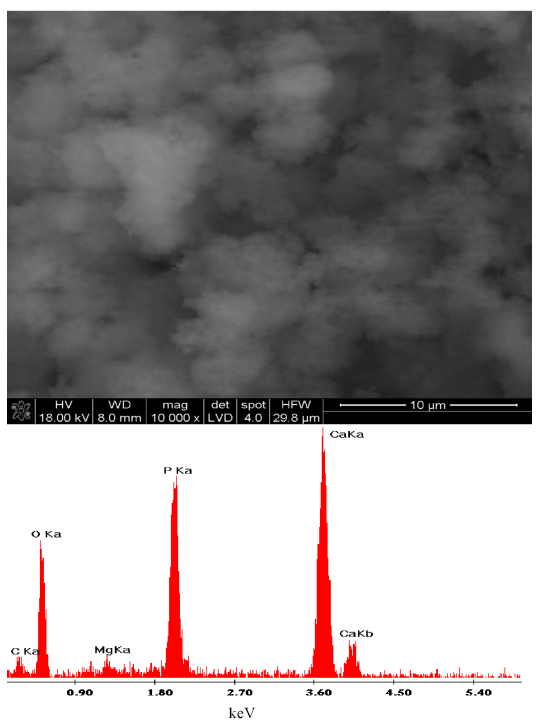

Fig. 6. As in Fig. 5, but for deposition time $90 \mathrm{~s}$.

that the best HAp deposition time is $90 \mathrm{~s}$ because in this case full surface was covered. Results of FTIR investigations were confirmed by SEM. Examples of SEM analysis are presented in Figs. 5 and 6.

Figure 5 presents SEM image (upper view) and X-ray spectrum (lower view) analysis of HAp layer deposited on Ti surface after $10 \mathrm{~s}$ of deposition time. In this case one can observe only Ag particles which agglomerate on the Ti surface. In opposite to Fig. 5, Fig. 6 which presents the surface modification after $90 \mathrm{~s}$ of HAp deposition, shows uniform coverage of the sublayer by hydroxyapatite.

\section{Summary}

- FTIR spectroscopy methods are particularly efficient tools for biomaterials analysis.

- Changes in phase composition of biomaterials can be determined by FTIR reflection technique based on FPA detection system. The spectra show characteristic bands due to $\mathrm{P}-\mathrm{O}$ vibrations of the $\mathrm{HAp}$ coatings films deposited on the Ti alloy.

- Results of FTIR imaging show that the untreated coatings do not contain phosphate groups, whereas electrophoresis process leads to the deposition of apatite with different thickness on alloys surfaces.

- Optical images and chemical maps of samples with phosphate layers obtained by EPD method allow to observe a correlation between the time deposition of nanohydroxyapatite and distribution of nanoparticles in the analyzed samples.

- The results obtained by FTIR method were confirmed by SEM technique. 


\section{Acknowledgments}

The research leading to these results has received funding from the European Community Seventh Framework Programme (FP7/2007-2013) under grant agreement no. 226716.

\section{References}

[1] K. Pravakaran, S. Kannan, S. Rajeswari, Trends Biomater. Artif. Organs 18, 114 (2005).

[2] E. Stodolak, C. Paluszkiewicz, M. Bogun, M. Blazewicz, J. Mol. Struct. 924, 208 (2009).

[3] C. Paluszkiewicz, A. Stoch, Vibrat. Spectrosc. 35, 183 (2004).

[4] I. Ozcan, H. Uysal, Dental Mater. 21, 773 (2005).

[5] J.C.M. Souza, R.M. Nascimento, A.E. Martinelli, Surf. Coat. Technol. 205, 787 (2010).

[6] A. Sugino, Ch. Ohtsuki, K. Tsuru, S. Hayakawa T. Nakano, Y. Okazaki, A. Osaka, Acta Biomater. 5, 298 (2009).

[7] O. Albayrak, O. El-Atwani, S. Altintas, Surf. Coat. Technol. 202, 2482 (2008).
[8] Ch. Lin, H. Han, F. Zhang, A. Li, J. Mater. Sci., Mater. Med. 19, 2567 (2008).

[9] B.H. Fellah, P. Layrolle, Acta Biomater. 5, 735 (2009).

[10] P. Innocenzi, T. Kidchob, L. Malfatti, S. Costacurta, M. Tajahashi, M. Piccinini, A. Marcelli, J. Sol-Gel Sci. Technol. 48, 253 (2008).

[11] C. Paluszkiewicz, A. Slosarczyk, D. Pijocha, M. Sitarz, M. Bućko, A. Zima, A. Chróścicka, M. Lewandowska-Szumieł, J. Mol. Struct. 976, 301 (2010).

[12] C. Paluszkiewicz, M. Blazewicz, J. Podporska, T. Gumuła, Vibrat. Spectrosc. 48, 263 (2008).

[13] M.E. Fleet, X. Liu, Biomaterials 28, 916 (2007).

[14] Q. Cheng, Ch. Li, V. Pavlinek, P. Saha, H. Wang, Appl. Surf. Sci. 252, 4154 (2006).

[15] N.I. Cade, T. Ritman-Meer, D. Richards, Phys Rev. B 79, 241404 (2009). 